\title{
Lebensqualität unter Active Surveillance
}

\begin{abstract}
—ür Männer mit Prostatakarzinom, die F eine Operation scheuen, ist Active Surveillance eine attraktive Option: Der invasive Eingriff wird aufgeschoben oder sogar ganz überflüssig, dennoch werden sie engmaschig überwacht. Niederländische Urologen untersuchten, wie sich diese Behandlungsstrategie im Vergleich zur Brachytherapie und der Robotergestützten laparoskopischen radikalen Prostatektomie auf die Lebensqualität der Patienten auswirkt. Für die retrospektive Analyse mussten 144 Patienten die validierten Fragebögen EORTC-QLQ-C30, EORTC-QLQ-PR25, IIEF-15 und ICIQSF ausfüllen. Nach der Behandlung waren die Lebensqualität-Scores für die drei Therapieoptionen weitgehen ähnlich. Patienten, die sich für Prostatektomie oder Brachytherapie entschieden hatten, hatten niedrigere Scores bei den physischen Subskalen wie sexuelle Funktion und Inkontinenz. Allerdings klagten auch 30 \% der Männer unter Active Surveillance über eine beeinträchtigte sexuelle Funktion.

Auch psychologisch ist Active Surveillance nicht frei von Nebenwirkungen.
\end{abstract}

Einer britischen Studie zufolge sind Männer unter Active Surveillance zweimal häufiger depressiv und leiden dreimal häufiger unter Angst als gesunde gleichaltrige Männer. Dies fanden die Forscher heraus, indem sie letztes Jahr 338 Prostatakarzinompatienten unter Active Surveillance zwei Fragebögen ausfüllen ließen: zum einen die Hospital Anxiety and Depression Scale (HADS) und zum anderen einen allgemeinen Bogen mit Angaben zu Alter, Beruf, Beziehungsstatus, Bildung und Ethnizität. Dr. Sam Watts, Southampton/UK, präsentierte die Auswertung der ersten 181 Patienten. Von diesen erfüllten 24 (14,9\%) die HADSKriterien für eine klinisch signifikante Depression und 36 (22,4\%) für Angst. Zum Vergleich: In der britischen Allgemeinbevölkerung über 65 betragen diese Werte $6 \%$ beziehungsweise $8 \%$. Eine Regressionsanalyse ergab ein erhöhtes Depressionsrisiko für Männer, die mit 15 oder früher die Schule abbrachen. Der Beziehungsstatus war wiederum ein signifikanter Prädiktor für Angst: Alleinstehende Männer hatten einen um 2,85 höheren Angst-Score als Verheiratete.
Durch das Aufschieben der Operation müssen viele Patienten im Laufe der Überwachung mehrfach biopsiert werden. Eine deutsch-kanadische Studie ging der Frage nach, ob das dazu führt, dass die postoperativen Ergebnisse dadurch schlechter ausfallen. Dazu werteten die Autoren die Daten von 10.743 Patienten aus der USamerikanischen Medicare-Datenbank aus, bei denen die Anzahl der Biopsien vor der radikalen Prostatektomie erfasst wurde. Tatsächlich hatten Männer, bei denen vor ihrer Operation zwei oder mehr Biopsien durchgeführt worden waren, häufiger eine erektile Dysfunktion (36,6\% vs. $31,3 \%$ ) und Harninkontinenz (31,4\% vs. $24,8 \%$ ) als Männer, die direkt operiert wurden. Das relative Risiko für eine erektile Dysfunktion war bei Männern mit zwei oder mehr Biopsien um $20 \%$ erhöht, das relative Harninkontinenzrisiko um $18 \%$. Dr. Florian Roghmann, Montréal, riet dazu, den Patienten vor der Therapieentscheidung über diese möglichen Folgen aufzuklären.

Sebastian Lux

\section{Urothelkarzinom des oberen Harntrakts: Risikofaktoren für Blasenkarzinomrezidiv}

B islang ist unklar, wie lange Patienten mit Harnleiter- und Nierenbeckentumoren nach einer Nephroureterektomie bei der Nachsorge zystoskopiert werden müssen. Ein schwedisches Review hatte zum Ziel, herauszufinden, wie lange nach der Operation des oberen Harntrakts ein Harnblasenkarzinom auftritt. Dazu werteten sie Daten von 614 Patienten aus, bei denen zwischen 1971 und 1998 ein Tumor des Harnleiters oder Nierenbeckens diagnostiziert wurde. 192 der Patienten $(31,3 \%)$ entwickelten im Verlauf ein Harnblasenkarzinom: 157 $(81,8 \%)$ bereits in den ersten beiden Jahren nach der Operation, 24 (12,5\%) in den Jahren drei bis fünf und elf Patienten $(5,7 \%)$ in den Jahren 5-20. Als signifikante Risikofaktoren stellten sich frühere
Blasentumoren, große Durchmesser des oberen Harntrakts und Carcinoma in situ des oberen Harntrakts heraus. Dr. Sten Hollmäng, Göteborg, der das Poster präsentierte, empahl daher kurze Zystoskopie-Intervalle in den ersten zwei Jahren nach der Nephroureterektomie, vor allem bei Risikopatienten. Nach fünf Jahren sollte man auf Zystoskopien verzichten.

Übereinstimmend dazu waren die Ergebnissen einer Studie aus Nordamerika und Europa - allerdings nur was die Rezidivhäufigkeit anging: Hier trat nach einem medianen Follow-up von 45 Monaten bei 577 von 1.839 Patienten (33\%) ein intravesikales Rezidiv auf. Als Risikofaktoren wurden dagegen neben früheren Blasentumoren biologisch hoch- aggressive Harnleiter- und Nierenbeckentumoren und die Art des Eingriffs (laparoskopischer Zugang und Präparation des distalen Harnleiters) identifiziert.

Auch eine japanische Arbeitsgruppe machte sich retrospektiv auf die Suche nach Risikofaktoren für ein Blasenkarzinomrezidiv. In ihre Analyse nahmen sie 754 Patienten auf, von denen 169 $(22 \%)$ nach einem medianen Follow-up von 41 Monaten ein Harnblasenkarzinom hatten. In dieser Studie waren papilläre Tumoren, Tumoren ohne Gefäßoder Lymphinvasion und ein fortgeschrittenes pathologisches Tumorstadium ( $\geq$ pT2) signifikant mit einem Blasenrezidiv assoziiert.

Sebastian Lux 